\title{
Comparing the effect of buprenorphine and methadone in the reduction of methamphetamine craving: a randomized clinical trial
}

\author{
Jamshid Ahmadi and Leila Razeghian Jahromi
}

\begin{abstract}
Background: We sought to test the effectiveness of methadone and buprenorphine in the treatment of methamphetamine withdrawal craving over a 17-day treatment period.

Methods: Patients were randomized into one of two groups. The study sample comprised 40 male subjects dependent on methamphetamine who met criteria of the Diagnostic and Statistical Manual of Mental Disorders, Fifth Edition, for methamphetamine dependence and withdrawal and were seeking treatment. Furthermore, they should have a history of daily methamphetamine use for at least 6 months and should have discontinued their use just before starting the protocol. Patients received $40 \mathrm{mg}$ of methadone or $8 \mathrm{mg}$ of buprenorphine per day and were treated in an inpatient psychiatric hospital. We used methamphetamine craving score, negative urine drug screening test (thin-layer chromatography) during the study, and retention in treatment.
\end{abstract}

Results: All 40 patients completed the study. Both drugs were effective in decreasing methamphetamine craving during methamphetamine withdrawal. Reduction of craving in the buprenorphine group was significantly more than in the methadone group $(P<0.05)$.

Conclusions: The results favor the efficacy and safety of buprenorphine as a short-term treatment for methamphetamine withdrawal craving. We should mention that it is to be expected that craving declines over time without any medication. Therefore, the conclusion may not be that methadone and buprenorphine both reduce the craving. Because buprenorphine is superior to methadone, only buprenorphine surely reduces craving.

Trial registration: Iranian Registry of Clinical Trials identifier: IRCT2015112125160N1. Registered on 4 June 2016.

Keywords: Methadone, Buprenorphine, Methamphetamine withdrawal craving

*Correspondence: jamshid_ahmadi@yahoo.com

Substance Abuse Research Center, Shiraz University of Medical Sciences,

Shiraz, Iran 


\section{Background}

Psychiatric disorders have been advancing problems in worldwide [1-4]. Among psychiatric problems, substance use disorders and substance-induced disorders, particularly those involving stimulants, are an increasing global concern [5-7]. In particular, methamphetamine use disorders and methamphetamine-induced psychiatric presentations to hospitals and outpatient centers are becoming increasingly problematic [8-11]. Using amphetamines can cause feelings of euphoria or irritability associated with increases in energy, wakefulness, concentration, and physical activity [7].

Abuse of methamphetamines is common. For example, in the United States, 18 million people over the age of 12 years have tried methamphetamines during their lives [12]. Similarly to other addictions, methamphetamine dependence is a lengthy, relapsing disorder. As part of a comprehensive treatment plan, medications may be required to prevent relapse. Prolonged consumption of methamphetamine can lead to abuse/dependence, aggression, violence, weight loss, impulsivity, decreased appetite, mood lability, poor concentration, hallucinations, delusions, and memory loss [12, 13].

In Iran, methamphetamine previously was illegally imported from other regions of the world (mainly the West), but now it is illegally provided and prepared in "underground" laboratories [10]. Currently, there is no standard of care, particularly medications, for the treatment of methamphetamine craving during methamphetamine withdrawal [7]. Buprenorphine and methadone are opioid medications that are widely used to treat opioid withdrawal symptoms, but, to our knowledge, they have not been used to treat methamphetamine withdrawal symptoms [7].

In this study, we examined buprenorphine and methadone as a way of treating craving during severe methamphetamine withdrawal. We theorized that the biochemistry and mechanisms of methamphetamine and opioid dependence are more or less similar because both drugs involve the endogenous opioid system [7, 10-16].

Substances such as methamphetamine, cocaine, and alcohol activate release of dopamine from cells originating in the brain's ventral tegmental area. It is a component of a neuronal circuit named the mesolimbic dopamine system and is joined to behavioral reward and motivation. Following exposure to alcohol, methamphetamine, or cocaine, dopamine released into the nucleus accumbens and prefrontal cortex strengthens and reinforces alcohol-, methamphetamine-, and cocaine-seeking behaviors $[17,18]$.

This trial is one of the first studies to provide data obtained by research comparing buprenorphine and methadone in the treatment of methamphetamine craving during methamphetamine withdrawal. The primary goal of this double-blind clinical trial was to test the effectiveness of $8 \mathrm{mg}$ of sublingual buprenorphine daily and $40 \mathrm{mg}$ of oral methadone daily in the treatment of methamphetamine withdrawal craving.

\section{Methods \\ Subjects}

Forty unpaid male subjects were recruited in 2016 and were diagnosed with severe methamphetamine dependence and withdrawal on the basis of Diagnostic and Statistical Manual of Mental Disorders, Fifth Edition (DSM-5), criteria by a board-certified psychiatrist using the Structured Clinical Interview for DSM-5, Clinical Version. We considered only males because the main psychiatric ward affiliated with the Shiraz University of Medical Sciences admits only male patients.

Prior to each interview, we explained the goals of the study, guaranteed confidentiality, and obtained written informed consent. The interviews and examinations were done on the premises of the treatment hospital because it appeared to be a nonthreatening and proper environment. Family members, friends, or relatives accompanied patients to the hospital. This attendance provided a condition in which we could verify the data and information obtained from the patients.

In addition to meeting DSM-5 criteria for methamphetamine use disorder, subjects required a history of daily methamphetamine use for at least 6 months and discontinuation of their use just before starting the trial. Patients were excluded from the trial if they had a primary diagnosis other than methamphetamine use disorder (dependency on substances other than methamphetamine) or major medical problems (cardiovascular, pulmonary, renal, or gastrointestinal diseases).

All patients provided written informed consent before entering into the trial. The research study was approved and monitored by the ethics committee of Shiraz University of Medical Sciences in adherence to the Declaration of Helsinki's ethical principles for medical research involving human subjects.

\section{Randomization}

In a double-blind manner, the patients were randomly assigned to one of the two treatment groups: buprenorphine or methadone. We employed a standard randomization procedure generated by computer to obtain a random sample set.

\section{Procedure}

The research staff were precisely trained and included an addiction psychiatrist, general psychiatrist, physician, psychologist, nurse, and statistician. The pills had the 
same shape and color. The patients and the research team were blinded to the medications for the duration of the trial. The ratings and interviews were performed by an adequately trained physician who was blinded to the medications and side effects. During the trial, no other intervention was allowed.

The principal investigator prepared a visual analogue scale (VAS) and verified it empirically for validity and reliability [10, 14-16]. We used the VAS to assess methamphetamine craving during methamphetamine withdrawal, with scores ranging from 0 to $10(0=$ no craving at all and $10=$ severe craving and temptation all the time). Moreover, we trained the subjects precisely and completely about scoring. In addition, a positive urine drug test for methamphetamine (thin-layer chromatography) before the beginning of the protocol and a negative urine drug test twice weekly during the study period were considered.

Consecutive patients were randomly assigned to receive either buprenorphine or methadone. Patients were randomly initiated on either $8 \mathrm{mg}$ of sublingual buprenorphine or $40 \mathrm{mg}$ of oral methadone daily. We followed the subjects for up to 17 days. Effectiveness was evaluated by daily interview and precise assessment of craving by asking the subjects about their experience.

\section{Statistical analysis}

Data analysis was carried out using PASW Statistics version 18 software (SPSS, Chicago, IL, USA). Student's $t$ test or the Mann-Whitney $U$ test was used to examine the differences in means. Chi-square analysis or Fisher's exact test was used to test for differences in frequencies. Repeated measures analysis of variance was used to examine the trends over time. All $P$ values were two-sided, and statistical significance was set at the $5 \%$ level.

\section{Results}

The Consolidated Standards of Reporting Trials (CONSORT) flowchart and checklist of patients in the trial are shown in Figs. 1 and 2. Forty-five patients were screened for this trial. Five patients were excluded because they did not meet the inclusion criteria. Of the 40 patients who were randomly allocated into one of the two groups, 20 patients were assigned to the methadone group and 20 patents were allocated to the buprenorphine group.

All of the 40 patients completed the 17-day trial. Of the 40 patients, $20(50 \%)$ received $8 \mathrm{mg}$ of buprenorphine and $20(50 \%)$ received $40 \mathrm{mg}$ of methadone. Table 1 displays the demographic characteristics of both groups.

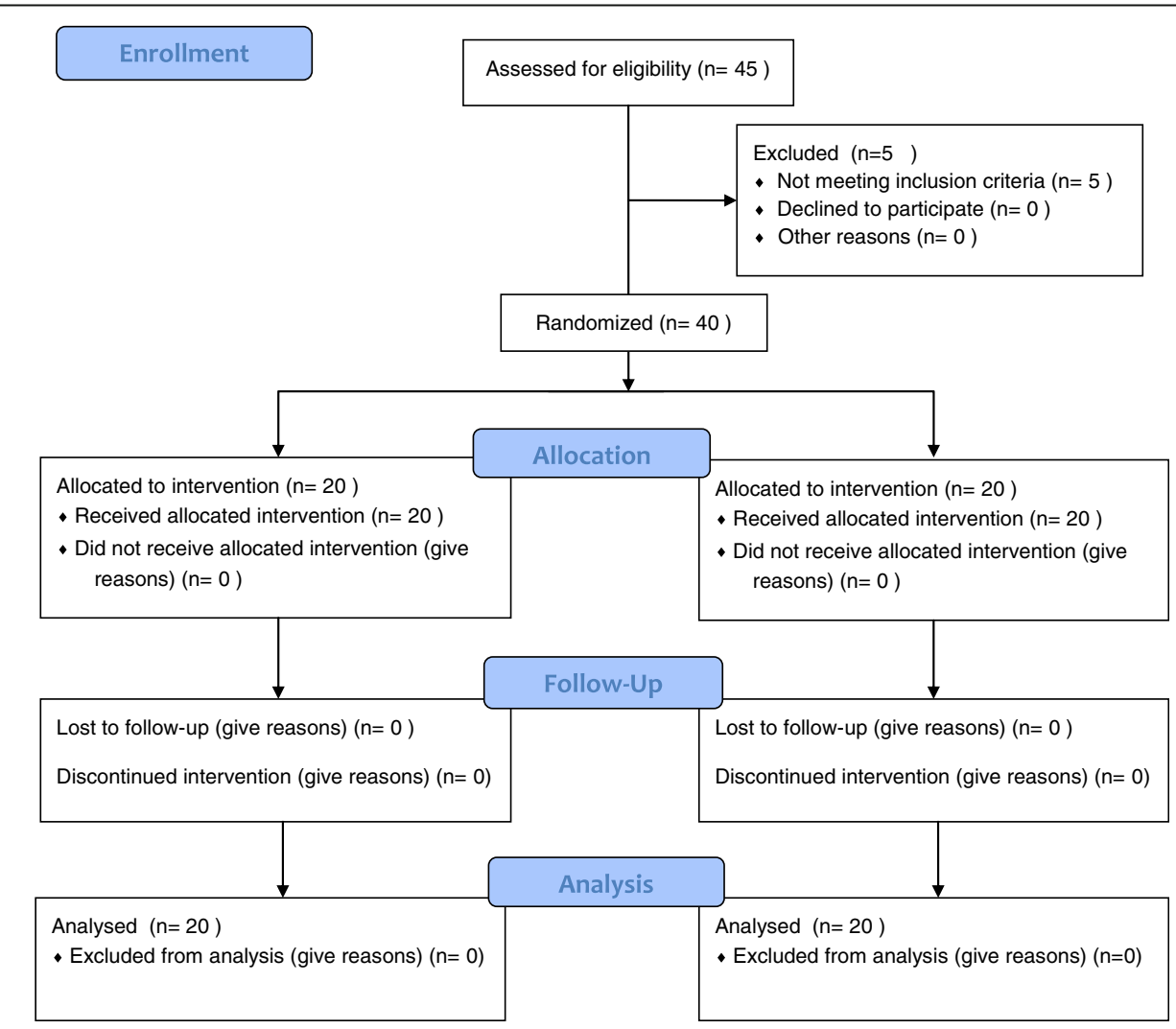

Fig. 1 Consolidated Standards of Reporting Trials (CONSORT) flowchart of the patients in this trial 


\section{CONSORT 2010 checklist of information to include when reporting a randomised trial*}

\begin{tabular}{|c|c|c|c|}
\hline Section/Topic & $\begin{array}{c}\text { Item } \\
\text { No }\end{array}$ & Checklist item & $\begin{array}{l}\text { Reported } \\
\text { on page No }\end{array}$ \\
\hline \multicolumn{4}{|l|}{ Title and abstract } \\
\hline & $1 \mathrm{a}$ & Identification as a randomised trial in the title & 1 \\
\hline & $1 \mathrm{~b}$ & Structured summary of trial design, methods, results, and conclusions (for specific guidance see CONSORT for abstracts) & 2 \\
\hline \multicolumn{4}{|l|}{ Introduction } \\
\hline Background and & $2 a$ & Scientific background and explanation of rationale & 4 \\
\hline objectives & $2 \mathrm{~b}$ & Specific objectives or hypotheses & 4 \\
\hline \multicolumn{4}{|l|}{ Methods } \\
\hline \multirow[t]{2}{*}{ Trial design } & $3 a$ & Description of trial design (such as parallel, factorial) including allocation ratio & 6 \\
\hline & $3 b$ & Important changes to methods after trial commencement (such as eligibility criteria), with reasons & 6 \\
\hline \multirow[t]{2}{*}{ Participants } & $4 a$ & Eligibility criteria for participants & 6 \\
\hline & $4 \mathrm{~b}$ & Settings and locations where the data were collected & 6 \\
\hline Interventions & 5 & $\begin{array}{l}\text { The interventions for each group with sufficient details to allow replication, including how and when they were } \\
\text { actually administered }\end{array}$ & 6 \\
\hline \multirow[t]{2}{*}{ Outcomes } & $6 a$ & $\begin{array}{l}\text { Completely defined pre-specified primary and secondary outcome measures, including how and when they } \\
\text { were assessed }\end{array}$ & 7 \\
\hline & $6 b$ & Any changes to trial outcomes after the trial commenced, with reasons & 7 \\
\hline \multirow[t]{2}{*}{ Sample size } & $7 a$ & How sample size was determined & 7 \\
\hline & $7 \mathrm{~b}$ & When applicable, explanation of any interim analyses and stopping guidelines & NA \\
\hline \multicolumn{4}{|l|}{ Randomisation: } \\
\hline \multirow{2}{*}{$\begin{array}{l}\text { Sequence } \\
\text { generation }\end{array}$} & $8 a$ & Method used to generate the random allocation sequence & 7 \\
\hline & $8 b$ & Type of randomisation; details of any restriction (such as blocking and block size) & 7 \\
\hline $\begin{array}{l}\text { Allocation } \\
\text { concealment } \\
\text { mechanism }\end{array}$ & 9 & $\begin{array}{l}\text { Mechanism used to implement the random allocation sequence (such as sequentially numbered containers), } \\
\text { describing any steps taken to conceal the sequence until interventions were assigned }\end{array}$ & 7 \\
\hline Implementation & 10 & $\begin{array}{l}\text { Who generated the random allocation sequence, who enrolled participants, and who assigned participants to } \\
\text { interventions }\end{array}$ & 7 \\
\hline \multirow[t]{2}{*}{ Blinding } & $11 \mathrm{a}$ & $\begin{array}{l}\text { If done, who was blinded after assignment to interventions (for example, participants, care providers, those } \\
\text { assessing outcomes) and how }\end{array}$ & 7 \\
\hline & $11 \mathrm{~b}$ & If relevant, description of the similarity of interventions & NA \\
\hline \multirow[t]{2}{*}{ Statistical methods } & $12 a$ & Statistical methods used to compare groups for primary and secondary outcomes & 8 \\
\hline & $12 \mathrm{~b}$ & Methods for additional analyses, such as subgroup analyses and adjusted analyses & 8 \\
\hline \multicolumn{4}{|l|}{ Results } \\
\hline \multirow{2}{*}{$\begin{array}{l}\text { Participant flow (a } \\
\text { diagram is strongly } \\
\text { recommended) }\end{array}$} & $13 a$ & $\begin{array}{l}\text { For each group, the numbers of participants who were randomly assigned, received intended treatment, and } \\
\text { were analysed for the primary outcome }\end{array}$ & 9 \\
\hline & $13 b$ & For each group, losses and exclusions after randomisation, together with reasons & 9 \\
\hline \multirow[t]{2}{*}{ Recruitment } & $14 a$ & Dates defining the periods of recruitment and follow-up & 9 \\
\hline & $14 \mathrm{~b}$ & Why the trial ended or was stopped & 9 \\
\hline Baseline data & 15 & A table showing baseline demographic and clinical characteristics for each group & 9 \\
\hline Numbers analysed & 16 & $\begin{array}{l}\text { For each group, number of participants (denominator) included in each analysis and whether the analysis was } \\
\text { by original assigned groups }\end{array}$ & 9 \\
\hline \multirow[t]{2}{*}{$\begin{array}{l}\text { Outcomes and } \\
\text { estimation }\end{array}$} & $17 a$ & $\begin{array}{l}\text { For each primary and secondary outcome, results for each group, and the estimated effect size and its } \\
\text { precision (such as } 95 \% \text { confidence interval) }\end{array}$ & 9 \\
\hline & $17 \mathrm{~b}$ & For binary outcomes, presentation of both absolute and relative effect sizes is recommended & 9 \\
\hline Ancillary analyses & 18 & $\begin{array}{l}\text { Results of any other analyses performed, including subgroup analyses and adjusted analyses, distinguishing } \\
\text { pre-specified from exploratory }\end{array}$ & 9 \\
\hline Harms & 19 & All important harms or unintended effects in each group (for specific guidance see CONSORT for harms) & 9 \\
\hline \multicolumn{4}{|l|}{ Discussion } \\
\hline Limitations & 20 & Trial limitations, addressing sources of potential bias, imprecision, and, if relevant, multiplicity of analyses & 10 \\
\hline Generalisability & 21 & Generalisability (external validity, applicability) of the trial findings & 11 \\
\hline Interpretation & 22 & Interpretation consistent with results, balancing benefits and harms, and considering other relevant evidence & 12 \\
\hline \multicolumn{4}{|l|}{ Other information } \\
\hline Registration & 23 & Registration number and name of trial registry & 13 \\
\hline Protocol & 24 & Where the full trial protocol can be accessed, if available & 13 \\
\hline Funding & 25 & Sources of funding and other support (such as supply of drugs), role of funders & 14 \\
\hline
\end{tabular}

*We strongly recommend reading this statement in conjunction with the CONSORT 2010 Explanation and Elaboration for important clarifications on all the items. If relevant, we also recommend reading CONSORT extensions for cluster randomised trials, non-inferiority and equivalence trials, non-pharmacological treatments, herbal interventions, and pragmatic trials. Additional extensions are forthcoming: for those and for up to date references relevant to this checklist, see www.consort-statement.org.

Fig. 2 Consolidated Standards of Reporting Trials (CONSORT) 2010 checklist of information to include when reporting a randomized trial 
Table 1 Demographic characteristics of the two study groups

\begin{tabular}{|c|c|c|c|c|c|}
\hline & & Methadone $(n=20)$ & Buprenorphine $(n=20)$ & Total & Significance \\
\hline Age, years, mean $\pm S D$ & & $31.2 \pm 9.04$ & $34.35 \pm 9.65$ & $32.78 \pm 9.37$ & $\begin{array}{l}t=1.065 \\
d f=38 \\
P=0.294\end{array}$ \\
\hline \multirow[t]{3}{*}{ Job, $n(\%)$} & Unemployed & $6(30)$ & $6(30)$ & $12(30)$ & \multirow{3}{*}{$\begin{array}{l}X^{2}=0.15 \\
P=0.928\end{array}$} \\
\hline & Self-employed & $9(45)$ & $8(40)$ & $17(42.5)$ & \\
\hline & Employee & $5(25)$ & $6(30)$ & $11(27.5)$ & \\
\hline \multirow[t]{2}{*}{ Marital status } & Single & $9(45)$ & $10(50)$ & $19(47.5)$ & \multirow[t]{2}{*}{$P=0.64$} \\
\hline & Married & $11(55)$ & $10(50)$ & $21(52.5)$ & \\
\hline \multirow[t]{4}{*}{ Education } & Illiterate & $5(25)$ & $5(25)$ & $10(25)$ & \multirow[t]{4}{*}{$P=1$} \\
\hline & Middle school & $9(45)$ & $9(45)$ & $18(45)$ & \\
\hline & High school & $4(20)$ & $4(20)$ & $8(20)$ & \\
\hline & Higher education & $2(10)$ & $2(10)$ & $4(10)$ & \\
\hline \multirow[t]{4}{*}{ Income (million Tooman) } & $<0.5$ & $2(10)$ & $3(15.8)$ & $5(12.5)$ & \multirow[t]{4}{*}{$P=0.736$} \\
\hline & $0.5<1$ & $16(80)$ & $12(63.2)$ & $28(70)$ & \\
\hline & $1<1.5$ & $1(5)$ & $3(15.8)$ & $4(10)$ & \\
\hline & $>1.5$ & $1(5)$ & $1(5.3)$ & $2(5)$ & \\
\hline
\end{tabular}

There were not any statistically significant differences between the two groups regarding age, education, employment, marital status, or income. According to Table 1 , the mean age of 40 methamphetamine dependents was 32.78 years (SD 9.37, range 21-55). The mean ages were 34.35 years (SD 9.65) for the buprenorphine group and 31.2 years (SD 9.04) for the methadone group. Table 2 indicates $t$ tests and analysis of variance with repeated measures for craving scores of both groups.

Figure 3 depicts our comparison of the mean craving between the two treatment groups.

On the basis of the data shown in Table 2 and Fig. 3, the methamphetamine craving score was reduced significantly in both the methadone and buprenorphine groups. (repeated measures analysis of variance; buprenorphine $F=125.572, P=0.000$; methadone $F=111.169$, $P=0.000)$.

All the patients had positive urine drug test results for methamphetamine at the beginning of the study. Furthermore, all the patients had negative urine drug tests for methamphetamine done twice weekly during the 17day study interval. During the trial, none of the patients developed significant side effects requiring treatment.

\section{Discussion}

To our knowledge, this is the first study to examine administration of methadone and buprenorphine for the treatment of methamphetamine craving during methamphetamine withdrawal. This study shows that although buprenorphine and methadone are both effective in treating methamphetamine craving during methamphetamine withdrawal, the craving in the buprenorphine group was significantly lower than that in the methadone group starting on the tenth day. Therefore, buprenorphine was more effective than methadone. It is to be expected that craving decreases over time without any medication. Thus, the conclusion cannot be drawn that methadone and buprenorphine both reduce the craving. Because buprenorphine is superior to methadone, only buprenorphine surely reduces the craving.

Patients in both groups did not report any significant side effects. Furthermore, we did not observe any side effects or complications related to buprenorphine or methadone. Besides, the cost considerations seem to be favorable, especially when we study the possibility of administration for outpatients without a need for hospitalization. We suggest these opioids as short-term inpatient treatments to enhance retention or even as long-term maintenance treatment to minimize relapse.

Opioid receptors, mainly the $\mu$ opioid receptor, a member of the opioid neuromodulatory system and of the large family of $G$ protein-coupled receptors, are the prominent pharmacological target for the treatment of moderate to severe pain and are of therapeutic value for the management of abuse of methamphetamines, opioids, cannabis, alcohol, and other drugs [19-29]. The mechanism of action by which opioids such as buprenorphine or methadone prevent or decrease methamphetamine craving and dependence is not fully understood; however, there are fundamental and basic interactions between the endogenous opioid neuropeptide systems and dopamine.

Naltrexone, which is an opioid antagonist, reduces and interrupts the interactions between dopamine and endogenous opioid neuropeptide systems [19-21]. We 
Table 2 Independent $t$ test and repeated measures analysis of variance for craving mean at 17 days in treatment groups

\begin{tabular}{|c|c|c|c|c|c|c|}
\hline & Buprenorphine $(n=20)$ & Methadone $(n=20)$ & $t$ value & $d f$ & $P$ value & Power \\
\hline Day 1 & $7 \pm 1.34$ & $7.2 \pm 1.28$ & 0.483 & 38 & 0.632 & 0.076 \\
\hline Day 2 & $6.05 \pm 1.76$ & $6.55 \pm 1.67$ & 0.921 & 38 & 0.363 & 0.147 \\
\hline Day 3 & $5.55 \pm 1.88$ & $6.3 \pm 1.92$ & 1.248 & 38 & 0.22 & 0.23 \\
\hline Day 4 & $4.6 \pm 1.93$ & $5.8 \pm 1.88$ & 1.991 & 38 & 0.054 & 0.493 \\
\hline Day 5 & $4.45 \pm 1.76$ & $5.45 \pm 2.11$ & 1.625 & 38 & 0.112 & 0.355 \\
\hline Day 6 & $3.85 \pm 1.79$ & $5 \pm 2.05$ & 1.891 & 38 & 0.066 & 0.454 \\
\hline Day 7 & $3.5 \pm 1.57$ & $4.6 \pm 2.14$ & 1.854 & 38 & 0.72 & 0.438 \\
\hline Day 8 & $2.9 \pm 1.59$ & $4.05 \pm 2.16$ & 1.917 & 38 & 0.063 & 0.463 \\
\hline Day 9 & $2.7 \pm 1.56$ & $3.7 \pm 2.05$ & 1.734 & 38 & 0.091 & 0.394 \\
\hline Day 10 & $2.3 \pm 1.34$ & $3.45 \pm 1.96$ & 2.166 & 38 & 0.37 & 0.558 \\
\hline Day 11 & $1.9 \pm 1.33$ & $3.05 \pm 1.79$ & 2.303 & 38 & 0.027 & 0.612 \\
\hline Day 12 & $1.5 \pm 1.32$ & $2.75 \pm 1.55$ & 2.746 & 38 & 0.009 & 0.764 \\
\hline Day 13 & $1.15 \pm 1.18$ & $2.45 \pm 1.23$ & 3.402 & 38 & 0.002 & 0.914 \\
\hline Day 14 & $1 \pm 1.03$ & $2 \pm 1.08$ & 3.008 & 38 & 0.005 & 0.832 \\
\hline Day 15 & $0.65 \pm 0.88$ & $1.6 \pm 1.27$ & - & - & $0.18^{a}$ & 0.763 \\
\hline Day 16 & $0.4 \pm 0.75$ & $1.4 \pm 1.23$ & - & - & $0.11^{\mathrm{a}}$ & 0.854 \\
\hline Day 17 & $0.15 \pm 0.37$ & $0.8 \pm 0.95$ & - & - & $0.35^{\mathrm{a}}$ & 0.783 \\
\hline$F$ & 125.572 & 111.169 & & & & \\
\hline$d f$ & 16 & 16 & & & & \\
\hline$P$ value & 0.000 & 0.000 & & & & \\
\hline Power & 1 & 0.947 & & & & \\
\hline Total of 17 days & $2.92 \pm 1.189$ & $3.89 \pm 1.517$ & 2.251 & 38 & 0.03 & 0.599 \\
\hline
\end{tabular}

${ }^{a}$ Mann-Whitney $U$ test

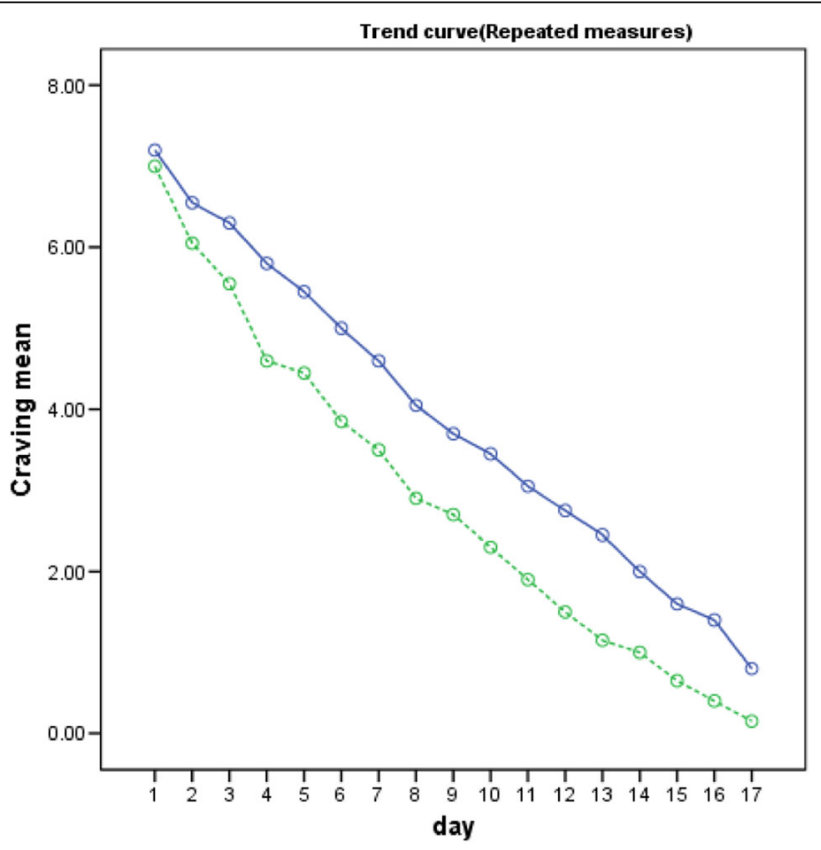

group

- methadone

- buprenorphine

Fig. 3 Comparison of mean craving between the two treatment groups 
theorized that opioid medications such as buprenorphine can enrich and improve the interactions between dopamine and endogenous opioid neuropeptide systems.

The findings of this research study are supportive of the effect of buprenorphine for the management of methamphetamine craving. There was superiority of buprenorphine compared with methadone $(P=0.03)$. We advise buprenorphine as short-term and inpatient treatment to increase retention or even as long-term maintenance treatment to reduce relapse.

\section{Limitations of the study}

Although we did not have a no-medication control group or a group treated with placebo in addition to the groups treated with buprenorphine and methadone, the fact that the two medications differed significantly in decrease of methamphetamine craving can compensate for this limitation; comparing the mean of craving between buprenorphine and methadone groups, there is a significant difference- $(P=0.03)$. We require a follow-up study to observe what happens when subjects are discharged from a controlled environment. It would be required to specify whether buprenorphine prevents short-term or long-term relapse.

\section{Conclusions}

The outcomes indicated a considerable reduction not only in the craving within each of the two groups but also between the groups. We believe that buprenorphine is a safe, effective, and valuable medication for decreasing methamphetamine craving during methamphetamine withdrawal and more effective than methadone. We recommend consideration of buprenorphine as a treatment for methamphetamine craving during methamphetamine withdrawal. It is to be expected that craving declines over time without any treatment. So, the conclusion cannot be that methadone and buprenorphine both decrease the craving. Because the buprenorphine is superior to methadone, only buprenorphine surely decreases the craving.

\section{Acknowledgements}

This study was supported by the Vice Chancellery for Research, Shiraz University of Medical Sciences, Shiraz, Iran (IR.SUMS.REC.1394.161, approved 13 December 2015).

\section{Availability of data and materials \\ Yes.}

\section{Authors' contributions}

JA proposed the idea for the research and wrote the proposal and the manuscript. LRJ collected the data and assisted with the writing of the manuscript. Both authors read and approved the final manuscript.

Authors' information

Not applicable.

\section{Competing interests}

The authors declare that they have no competing interests.

\section{Consent for publication}

Yes.

\section{Ethics approval and consent to participate}

All the patients provided written informed consent, and the study was approved and monitored by the ethics committee of Shiraz University of Medical Sciences to ensure that it adhered to the Declaration of Helsinki ethical principles for medical research involving human subjects

(IR.SUMS.REC.1394.161, approved 13 December 2015).

\section{Publisher's Note}

Springer Nature remains neutral with regard to jurisdictional claims in published maps and institutional affiliations.

Received: 10 March 2017 Accepted: 22 May 2017

Published online: 06 June 2017

\section{References}

1. Ahmadi J, Ahmadi N, Soltani F, Bayat F. Gender differences in depression scores of Iranian and German medical students. Iran J Psychiatry Behav Sci. 2014:8(4):70-3.

2. Mackay-Smith M, Ahmadi J, Pridmore S. Suicide in shooting galleries. ASEAN J Psychiatry. 2015;16(1):50-6.

3. Ahmadi J, Fakoor A, Pezeshkian P, Khoshnood R, Malekpour A. Substance use among Iranian psychiatric inpatients. Psychol Rep. 2001;89:363-5.

4. Ahmadi J, Ahmed MG. Dubai medical college students' attitudes towards substance use. J Addict Res Ther. 2013;S6:005. doi:10.4172/2155-6105.S6-005.

5. Ahmadi J, Kampman KM, Osline DM, Pettinati HM, Dackis C, Sparkman T. Predictors of treatment outcome in outpatient cocaine and alcohol dependence treatment. Am J Addict. 2009:18(1):81-6.

6. Ahmadi J, Kampman K, Dackis C, Sparkman T, Pettinati H. Cocaine withdrawal symptoms identify type B cocaine-dependent patients. Am J Addict. 2008;17(1):60-4.

7. Sadock BJ, Sadock VA, Ruiz P. Substance use and addictive disorders. In: Sadock BJ, Sadock VA, Ruiz P, editors. Kaplan \& Sadock's synopsis of psychiatry. 11th ed. Philadelphia: Lippincott Williams \& Wilkins; 2015. p. 616-93.

8. Ahmadi J, Keshtkar M, Pridmore S. Methamphetamine induced synesthesia: a case report. Am J Addict. 2011;20(3):306.

9. Ahmadi J, Sahraian A, Dastgheib SA, Mowla A, Ahmadzadeh L. Management of methamphetamine-induced psychosis by 8 sessions of ECT. Scholars J Appl Med Sci. 2015;3(3H):1565-6.

10. Ahmadi J. The effect of buprenorphine and bupropion in the treatment of methamphetamine dependency and craving. Br J Med Med Res. 2015;10(2):1-4.

11. Ahmadi J, Pridmore S, Ekramzadeh S. Successful use of electro convulsive therapy in the management of methamphetamine induced psychosis with onset during intoxication. J Addict Depend. 2015;1(1):1-2.

12. Hoffman WF, Moore M, Templin R, McFarland B, Hitzemann RJ, Mitchell SH. Neuropsychological function and delay discounting in methamphetaminedependent individuals. Psychopharmacology (Berl). 2006;188(2):162-70.

13. Salo R, Nordahl TE, Natsuaki Y, Leamon MH, Galloway GP, Waters C, et al. Attentional control and brain metabolite levels in methamphetamine abusers. Biol Psychiatry, 2007:61(11):1272-80.

14. Ahmadi J. Methylphenidate in the treatment of methamphetamine withdrawal craving: a novel outcome. J Drug Abuse. 2016;2(1):12.

15. Ahmadi J. Fast treatment of methamphetamine related anxiety and depressive disorders: a novel approach. J Addict Med Ther Sci. 2016;1 (2): 044-6. doi:10.17352/2455-3484.000011.

16. Ahmadi J. Recurrent psychosis related to methamphetamine. J Harmoniz Res Med Health Sci. 2016;3(1):51-5.

17. Lovinger DM, Roberto M. Synaptic effects induced by alcohol. Curr Top Behav Neurosci. 2013;13:31-86.

18. Ray LA, Chin PF, Miotto K. Naltrexone for the treatment of alcoholism: clinical findings, mechanisms of action, and pharmacogenetics. CNS Neurol Disord Drug Targets. 2010;9:13-22. 
19. Manglik A, Kruse AC, Kobilka TS, Thian FS, Mathiesen JM, Sunahara RK, et al. Crystal structure of the micro-opioid receptor bound to a morphinan antagonist. Nature. 2012;485:321-6.

20. Huang W, Manglik A, Venkatakrishnan AJ, Laeremans T, Feinberg EN, Sanborn AL, et al. Structural insights into micro-opioid receptor activation. Nature. 2015;524:315-21.

21. Kaserer T, Lantero A, Schmidhammer H, Spetea M, Schuster D. $\mu$ Opioid receptor: novel antagonists and structural modeling. Sci Rep. 2016;6:21548.

22. Ahmadi J. Hashish-induced olfactory hallucination: a novel finding. J Psychiatry. 2015;18:330. doi:10.4172/2378-5756.1000330.

23. Ahmadi J. Positive effect of low dose of buprenorphine in the treatment of severe hashish withdrawal craving: an original arcade. J Addict Depend. 2016;2(2):1-3.

24. Ahmadi J. Cannabis induced psychosis treatment: an original approach. J Addict Depend. 2016;2(2):1-2.

25. Ahmadi J. The effect of buprenorphine on the reduction of cannabis and heroin craving and suicidal thoughts: a new finding. Insights Biomed. 2016; $1: 2$

26. Ahmadi J. Alcohol withdrawal craving treatment with low dose of buprenorphine: a new experience. J Psychiatry. 2016;19:387. doi:10.4172/ 2378-5756.1000387.

27. Ahmadi J. Treatment of cannabis related psychosis with electroconvulsive therapy (ECT): a rapid approach. J Harmoniz Res Med Health Sci. 2016;3(1): 44-50.

28. Pasternak GW. Opiate pharmacology and relief of pain. J Clin Oncol. 2014; 32:1637-9

29. Spetea M, Asim MF, Wolber G, Schmidhammer H. The $\mu$ opioid receptor and ligands acting at the $\mu$ opioid receptor, as therapeutics and potential therapeutics. Curr Pharm Des. 2013;19:7415-34.

\section{Submit your next manuscript to BioMed Central and we will help you at every step:}

- We accept pre-submission inquiries

- Our selector tool helps you to find the most relevant journal

- We provide round the clock customer support

- Convenient online submission

- Thorough peer review

- Inclusion in PubMed and all major indexing services

- Maximum visibility for your research

Submit your manuscript at www.biomedcentral.com/submit 